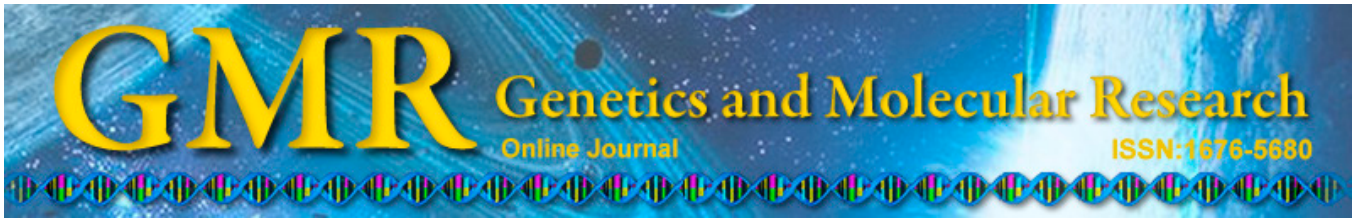

\title{
Characterization and significance of MUC1 and c-myc expression in elderly patients with papillary thyroid carcinoma
}

\author{
Y.-J. Hu ${ }^{1}$, X.-Y. Luo ${ }^{2}$, Y. Yang ${ }^{3}$, C.-Y. Chen ${ }^{1}$, Z.-Y. Zhang ${ }^{4}$ and X. Guo ${ }^{1}$ \\ ${ }^{1}$ Department of Head and Neck Surgery, Tangshan Gongren Hospital, \\ Tangshan, Hebei Province, China \\ ${ }^{2}$ Emergency Department, Kailuan General Hospital, Tangshan, \\ Hebei Province, China \\ ${ }^{3}$ Department of Endocrinology, Tangshan Gongren Hospital, Tangshan, \\ Hebei Province, China \\ ${ }^{4}$ Department of Pathology, Tangshan Gongren Hospital, Tangshan, \\ Hebei Province, China \\ Corresponding author: Z.-Y. Zhang \\ E-mail: zhangzhiy868@126.com
}

Genet. Mol. Res. 14 (4): 15325-15330 (2015)

Received January 11, 2015

Accepted June 8, 2015

Published November 30, 2015

DOI http://dx.doi.org/10.4238/2015.November.30.9

\begin{abstract}
The aim of this study was to examine the expression of mucin 1 (MUC1) and c-myc and the significance thereof in elderly patients with papillary thyroid carcinoma. The expression levels of MUC1 and c-myc were examined by immunohistochemical methods in 58 patients with papillary thyroid carcinoma, 35 with nodular goiter, and 30 subjects with normal thyroid tissue. The positive rate of MUC1 detection in papillary thyroid carcinoma was $77.6 \%(45 / 58)$, while it was $90.0 \%(9 / 10)$ in those with infiltration and $88.2 \%(15 / 17)$ in those with lymphatic metastasis. The positive rate of c-myc was $81.0 \%$ $(47 / 58)$ in those with papillary thyroid carcinoma and $100.0 \%(17 / 17)$ in those with lymphatic metastasis. These results demonstrated that there were differences in MUC1 and c-myc expression in benign and papillary thyroid carcinoma and that these differences were related to
\end{abstract}


thyroid cancer lymphatic metastasis.

Key words: Carcinoma papillary; Thyroid; Mucin 1; C-myc; Proto-oncogene proteins; Immunohistochemistry

\section{INTRODUCTION}

Thyroid cancer is the most common endocrine malignancy, of which papillary thyroid carcinoma (PTC) is the most common histological type, accounting for approximately $78 \%$ of cases. In recent years, the incidence of PTC in elderly patients has significantly increased, with an annual rate of increase of $4 \%$ (Yang et al., 2012), and most patients have a poor prognosis (Zhao et al., 2009). However, the pathogenesis of PTC is still not very clear, and further studies are necessary in order to explore the relevance genes thought to be closely associated with incidence and developmental mechanisms of PTC. In this study, we utilized immunohistochemistry to detect the presence of expression of mucin 1 (MUC1) and c-myc in human PTC, nodular goiter (NG), and normal thyroid tissue (NTT). We further discuss the relationships of our results with the biological behaviors of these proteins, in order to lay the foundation for further study on the relationship of MUC1, c-myc, and thyroid tumors.

\section{MATERIAL AND METHODS}

\section{Materials}

All samples utilized were paraffin-embedded specimens obtained from patients who had nodular goiter or papillary carcinoma, had been admitted to Tangshan Worker's Hospital from January 2011 to June 2013, and were over 60 years of age. The patients included 58 individuals who had PTC confirmed by pathologic examination and surgical resection (13 men and 45 women, with an average age of 68.16 years), and 35 with $\mathrm{NG}$ diagnosed by pathologic examination and surgical resection (8 men and 27 women, with an average age of 67.84 years). As controls, 30 patients with NG diagnosed by pathologic examination and surgical resection were recruited, including 7 men and 23 women with a mean age of 67.92 years, and the adjacent NTT (distance to tumor $>2 \mathrm{~cm}$ ) was selected. All specimens were confirmed by hematoxylin and eosin (H\&E) staining.

\section{Detection methods}

Paraffin-embedded tissue was consecutively cut to obtain 4- $\mu$ m slices; high-pressure thermal repair was used, and the slices were stained using the labeled streptavidin binding (LSAB) immunohistochemical staining method. The MUC1 monoclonal antibody was purchased from the American Diagnostica Company (Stamford, CT, USA); mouse anti-human cmyc mAb and LSAB kits were purchased from Dako Corporation (Glostrup, Denmark). The immunohistochemical procedure was performed in strict accordance with the requirements of the kit. After baking at $65^{\circ} \mathrm{C}$ for $4 \mathrm{~h}$ and immersion in $3 \% \mathrm{H}_{2} \mathrm{O}_{2}$ for $15 \mathrm{~min}, 0.01 \mathrm{mM}$ citrate buffer, $\mathrm{pH}$ 6.0, was used for microwave antigen retrieval at $100^{\circ} \mathrm{C}$ for $20 \mathrm{~min}$. Then, the sample was cooled to room temperature and washed with phosphate-buffered saline (PBS). Following incubation with calf serum for $15 \mathrm{~min}$, the first antibody was added dropwise and the sample incubated at $4^{\circ} \mathrm{C}$ for $12 \mathrm{~h}$. Subsequently, the sample was washed three times in PBS for $5 \mathrm{~min}$ each, and a solution of polymer enhancers was added, followed by incubation at room tempera- 
ture for $30 \mathrm{~min}$. The sample was then washed with PBS, stained with 3,3' diaminobenzidine, and re-stained with hematoxylin. PBS was used instead of the first antibody as a negative control.

\section{Determination of results}

Stained sections were observed under a microscope; brown color indicated positive staining. Five high-power fields (400X) in each case were randomly selected and graded by the percentage of positive cells. The determination of MUC1 was in accordance with the criteria of Yuan et al. (2003): Fields with a percentage of positive cells less than $20 \%$ were scored as negative (-), and a percentage of positive cells greater than or equal to $20 \%$ was scored as positive $(+)$. According to the standards reported by Lu et al. (2004), the results for c-myc were determined as follows: fields with a percentage of positive cells less than $10 \%$ were scored as negative (-), and those with a percentage of positive cells no less than $10 \%$ were scored as positive $(+)$.

\section{Statistical methods}

The SPSS13.0 statistical software (SPSS, Chicago, IL, USA) was used for statistical analysis. Measurement data of two groups were compared using a $t$-test, and those of multiple groups were compared using analysis of variance; count data were compared using the $\chi^{2}$ test. $\mathrm{P} \leq 0.05$ was considered to be statistically significant.

\section{RESULTS}

\section{General comparison of patients with PTC, NG, and NTT}

In this study, 58 patients with PTC, 35 with NG, and 30 with NTT were selected; no statistical difference was found in age, gender distribution, and the maximum diameter of the tumor among the three groups $(\mathrm{P}>0.05)$ (Table 1).

\begin{tabular}{|c|c|c|c|c|c|}
\hline \multirow[t]{2}{*}{ Clinical features } & \multicolumn{3}{|c|}{ Patients } & \multirow[t]{2}{*}{$\chi^{2}$ value/F value } & \multirow[t]{2}{*}{$P$ value } \\
\hline & PTC & NG & NTT & & \\
\hline \multicolumn{6}{|l|}{ Gender } \\
\hline Male & 13 & 8 & 7 & 0.001 & 0.995 \\
\hline Female & 45 & 27 & 23 & & \\
\hline Age (years) & $68.16 \pm 2.56$ & $67.84 \pm 2.92$ & $67.92 \pm 3.12$ & 0.160 & 0.850 \\
\hline \multicolumn{6}{|l|}{ Nodule size $(\mathrm{cm})$} \\
\hline (Maximum diameter) & $1.23 \pm 0.60$ & $1.25 \pm 0.53$ & $1.28 \pm 0.62$ & 0.07 & 0.930 \\
\hline
\end{tabular}

\section{Expression of MUC1 and c-myc in patients with PTC, NG, or NTT}

MUC1 expression was primarily localized in the cytoplasm; the positive rates of MUC1 in patients with NTT, NG, and PTC were 10.0 (3/30), 22.9 (8/35), and 77.6\% (45/58), respectively, with an obvious trend of increase with increase in clinical severity. These differences were statistically significant $(\mathrm{P}<0.01)$, indicating that there were significant differences in MUC1 expression between normal thyroid tissue and benign and malignant tumors $\left(\chi^{2}=\right.$ $\left.36.429, \mathrm{P}<0.01, \chi^{2}=26.673, \mathrm{P}<0.01\right)($ Table 2). 
Table 2. MUC1 and c-myc expression in different patients.

\begin{tabular}{|c|c|c|c|c|c|c|c|c|c|}
\hline \multirow[t]{2}{*}{ Clinical features } & \multirow[t]{2}{*}{ Patients (N) } & \multicolumn{2}{|c|}{ MUC1 } & \multirow[t]{2}{*}{$\chi^{2}$ value } & \multirow[t]{2}{*}{ P value } & \multicolumn{2}{|c|}{ c-myc } & \multirow[t]{2}{*}{$\chi^{2}$ value } & \multirow[t]{2}{*}{$\mathrm{P}$ value } \\
\hline & & - & + & & & - & + & & \\
\hline PTC & 58 & 13 & - & 46.558 & 0.000 & 11 & 47 & 32.049 & 0.000 \\
\hline NG & 35 & 27 & 8 & & & 19 & 16 & & \\
\hline NTT & 30 & 27 & 3 & & & 24 & 6 & & \\
\hline
\end{tabular}

$\mathrm{PTC}=$ papillary thyroid carcinoma; $\mathrm{NG}=$ nodular goiter; $\mathrm{NTT}=$ normal thyroid tissue.

c-myc expression was primarily localized in the nucleus. Expression was mostly positive in the PTC samples with a positive rate of $81.0 \%(47 / 58)$; the positive rate was $45.7 \%(16 / 35)$ in patients with NG, but expression was mostly negative in NTT samples, with a positive rate of $20.0 \%(6 / 30)$. There were significant differences in the positive rates of the three groups $(\mathrm{P}<0.01)$. c-myc expression in PTC was more extensive than that in NG $\left(\chi^{2}=12.461, \mathrm{P}<0.01\right)$, and the expression in NG was stronger than that in NTT $\left(\chi^{2}=4.770, \mathrm{P}<0.05\right)$.

\section{Relationships of MUC1 and c-myc expression with the clinical features of the 58 patients with PTC}

Positive rates of MUC1 expression in patients with PTC with local invasion and non-local infiltration were respectively $90.0(9 / 10)$ and $54.2 \%(26 / 48)$, with a statistically significant difference $(\mathrm{P}<0.05)$. Positive expression rates of patients with and without lymph node lymphatic metastasis were $88.2(15 / 17)$ and $53.7 \%(22 / 41)$, respectively, and this difference was also statistically significant $(\mathrm{P}<0.05)$. MUC1 expression was independent of the gender, age, and tumor size of patients with PTC $(\mathrm{P}>0.05)$, but was related to local invasion and lymph node metastasis. The positive expression rates of MUC1 in patients with local tumor invasion and lymph node lymphatic metastasis were higher than those in patients without local infiltration and lymph node lymphatic metastasis $(\mathrm{P}<0.05)$ (Table 3).

Table 3. Correlation of MUC1 and c-myc expression with the clinical features of 58 patients with PTC.

\begin{tabular}{|c|c|c|c|c|c|c|c|c|c|}
\hline \multirow[t]{2}{*}{ Clinical features } & \multirow[t]{2}{*}{ Patients } & \multicolumn{2}{|c|}{ MUC1 } & \multirow[t]{2}{*}{$\chi^{2} / t$ value } & \multirow[t]{2}{*}{ P value } & \multicolumn{2}{|c|}{ c-myc } & \multirow[t]{2}{*}{$\chi^{2} / t$ value } & \multirow[t]{2}{*}{$P$ value } \\
\hline & & - & + & & & - & + & & \\
\hline \multicolumn{10}{|l|}{ Gender } \\
\hline Male & 13 & 2 & 11 & 0.476 & 0.490 & 3 & 10 & 0.314 & 0.575 \\
\hline Female & 45 & 11 & 34 & & & 14 & 31 & & \\
\hline Age (years) & - & $67.42 \pm 2.12$ & $68.56 \pm 2.07$ & 1.637 & 0.107 & $67.95 \pm 1.98$ & $68.31 \pm 2.07$ & 0.564 & 0.575 \\
\hline \multicolumn{10}{|l|}{ Tumor size } \\
\hline$<1 \mathrm{~cm}$ & 34 & 8 & 26 & 2.230 & 0.328 & 10 & 24 & 0.490 & 0.783 \\
\hline$>1 \mathrm{~cm}$ & & & & & & & & & \\
\hline$<4 \mathrm{~cm}$ & 16 & 7 & 9 & & & 6 & 10 & & \\
\hline$>4 \mathrm{~cm}$ & 8 & 2 & 6 & & & 2 & 6 & & \\
\hline \multicolumn{10}{|l|}{ Local invasion } \\
\hline With & 10 & 1 & 9 & 4.441 & 0.035 & 2 & 8 & 0.506 & 0.477 \\
\hline Without & 48 & 22 & 26 & & & 15 & 33 & & \\
\hline \multicolumn{10}{|c|}{ Lymph node metastasis } \\
\hline With & 17 & 2 & 15 & 6.220 & 0.013 & 0 & 17 & 6.274 & 0.012 \\
\hline Without & 41 & 19 & 22 & & & 12 & 29 & & \\
\hline
\end{tabular}

$\mathrm{PTC}=$ papillary thyroid carcinoma 
c-myc expression was only found to be associated with lymph node metastasis ( $\mathrm{P}<$ $0.05)$, and the positive rate was $100.0 \%$ in patients with lymphatic metastases (17/17); however, it had no correlation with other clinical features $(\mathrm{P}>0.05)$ (Table 3$)$.

\section{DISCUSSION}

MUC1 is an important cancer-associated antigen gene and plays an important role in the incidence and development of malignant tumors. Because of the high expression of MUC1, the distribution of polarity is destroyed, and the adhesion between tumor cells is reduced, resulting in the adhesion of tumor and normal cells, immune escape, tumor cell proliferation, tumor invasion, and lymphatic metastasis (Peng et al., 2001). Many studies on MUC1 have focused on its role in breast cancer; however, few studies that relate have been published. to thyroid cancer c-myc is a common proto-oncogene, which can promote cell proliferation and induce cell apoptosis (Yang et al., 2008), and plays a role in regulating cell growth, differentiation, and malignant transformation. In particular, offset or excessive c-myc expression plays an important role in thyroid cancer development (Salvatore et al., 1996).

This study found that the positive rates of MUC1 and c-myc protein expression in cancer tissues from patients with PTC were significantly higher than those in patients with NTT and $\mathrm{NG}$, which indicated that there were obvious differences in their expression in benign and malignant thyroid lesions. Therefore, it might be considered that MUC1 and C- myc can not only serve as a reference indicator of thyroid cancer in PTC, but might also play a role in the differential diagnosis of thyroid cancer and benign thyroid lesions. Furthermore, MUC1 expression in patients with local invasion and lymph node metastasis was also shown to be higher than that in patients without invasion and lymphatic metastasis, suggesting that MUC1 played a role in promoting the invasion and lymphatic metastasis of thyroid cancer; this finding is consistent with the results of Imai et al. (2010). We also found that c-myc expression was related only to lymph node metastasis; therefore, it might be considered as a basis to determine the likelihood of poor prognosis. Together, these results suggest that the detection of MUC1 and c-myc expression in patients would be helpful in the determination of the biological behavior of thyroid cancer and the correct prediction of prognosis, which can be used to guide clinical treatment.

\section{Conflicts of interest}

The authors declare no conflict of interest.

\section{REFERENCES}

Imai K, Hayashi T, Suwa T, Makiguchi Y, et al. (2010). MUC1 as a human tumor marker. Intl. Congress Series 1223: $125-133$

Lu XB, An ZF, Wang QZ, Yin DT, et al. (2004). Expression of survivin and c-myc in differentiated thyroid carcinoma and clinical significance. Chin. J. Exp. Surg. 21: 705-706.

Peng YF, Cai YH and Zhong H (2001). The structure, function and application of MUC1. Lett. Biotechnol. 12: 130-139.

Salvatore D, Celetti A, Fabien N, Paulin C, et al. (1996). Low frequency of p53 mutations in human thyroid tumours; p53 and Ras mutation in two out of fifty-six thyroid tumours. Eur. J. Endocrinol. 134: 177-183.

Yang JJ, Yao J, Shen FQ, Chen Jiayu, et al. (2012). The relationship of Notch-1 signaling pathway with human papillary carcinoma of thyroid. Chin. J. Cell Biol. 34: 120-126. 
Yang LP, Feng J and Li N (2008). Study on the expression of oncogene c-myc in thyroid tissure of patients with Graves disease and papillary thyroid carcinoma. J. North China Coal Medic. Coll. 10: 735-737.

Yuan SF, Li KZ and Wang L (2003). Expression of MUC1 in thyroid cancer and benign thyroid diseases and its clinical significance. Chin. J. Gen. Surg. 18: 488-490.

Zhao HD, Gong L, Zhang J, Li Y, et al. (2009). Expression analysis of ndrg2 and c-myc in human thyroid tumors by utilize thyroid tissue arrays. Prog. Mod. Biomed. 9: 4605-4608. 